Article

\title{
Chemical Profiles of Cultivated Agarwood Induced by Different Techniques
}

\author{
Tingting Yan ${ }^{1}$, Sheng Yang ${ }^{2}$, Yuan Chen ${ }^{2}$, Qian Wang ${ }^{2}$ and Gaiyun Li ${ }^{2, *}$ \\ 1 Research Institute of Forestry New Technology, Chinese Academy of Forestry, Beijing 100091, China; \\ yantt@criwi.org.cn \\ 2 Research Institute of Wood Industry, Chinese Academy of Forestry, Beijing 100091, China; \\ yangsheng@criwi.org.cn (S.Y.); chenyuan@criwi.org.cn (Y.C.); 15501130520@163.com (Q.W.) \\ * Correspondence: ligy@criwi.org.cn; Tel.: +86-010-6288-9433
}

Academic Editor: Maria Carla Marcotullio

Received: 1 April 2019; Accepted: 9 May 2019; Published: 24 May 2019

check for updates

\begin{abstract}
Agarwood is the resinous wood produced in some Aquilaria species and is highly valued for wide usages in medicine, incense, and perfume. To protect the threatened Aquilaria species, the cultivation of Aquilaria sinensis and artificial agarwood induction techniques have been effectively established in China. To evaluate the quality of agarwood induced by different techniques, patterns of chemical constituents in artificial agarwood by four methods (wounding using an axe, burning-chisel-drilling, chemical inducer, and biological inoculation) were analyzed and compared by UPLC-ESI-MS/MS and GC-EI-MS in this study. Results of GC-MS gave a panorama of chemical constituents in agarwood, including aromatic compounds, steroids, fatty acids, sesquiterpenoids, and 2-(2-phenlyethyl)-chromones (PECs). Sesquiterpenoids were dominant in agarwood induced by wounding using an axe. PEC comprised over $60 \%$ of components in agarwood produced by biological inoculation and chemical inducers. PECs were identified by UPLC-ESI-MS/MS in all artificial agarwood and the relative contents varied in different groups. Tetrahydro-2-(2-phenylethyl)-chromones (THPECs) in wounding by axes induced agarwood were lower while 2-(2-phenylethyl)-chromones (FPECs) were higher than other groups. The results showed that methods used for inducing agarwood formation in Aquilaria sinensis affect the chemical constituents of agarwood.
\end{abstract}

Keywords: cultivated agarwood; Aquilaria sinensis; 2-(2-phenylethyl)-chromone; sesquiterpenoid

\section{Introduction}

The process of agarwood formation is initiated in the parenchyma cells in some species belonging to Thymelaeaceae family [1]. The cells activate protective machinery to synthesize secondary metabolites which defend against the stress [2]. The chemicals are produced, stored, and distributed in the wounded areas, filling the anatomical compartments [3]. Based on the process, various artificial agarwood induction methods have been invented and classified into two groups: conventional and nonconventional methods [4]. The conventional techniques include physical intrusions such as burning-chisel-drilling and wounding using an axe, which have been established for nearly 1000 years in ancient China [5]. Non-conventional methods have been newly developed in recent years [4]. Solvents containing ions or some microbes are injected into trunks of health trees to stimulate agarwood formation [6,7]. These methods are intensively practiced in plantations of China and other agarwood producing countries [5]. Agarwood produced by artificial induction techniques will inevitably be the majority in the commercial and medical market in the near future. Therefore, the quality of cultivated agarwood is of great concern. Some recent reports suggested that agarwood induced by nonconventional methods were comparable with wild agarwood while others reported the obvious differences in chemical constituents between wild and cultivated agarwood [8-10]. Studies 
focusing on agarwood yielded by conventional induction methods are still few [11]. Analysis and comparison of chemical constituents in agarwood induced by different methods is obligatory to provide comprehensive assessments of them.

More and more researchers are beginning to engage in illustrating the mechanisms behind secondary metabolites synthesis during agarwood formation for better performance of artificial agarwood techniques $[4,12,13]$. Chromones are detectable during the first 20 days after treatment of chemical inducers [14]. Major chromones will all appear and proportions become steady after nine months [15]. The results suggested formation of agarwood is complex and dynamic. Whether induction methods affect chemical constituents in agarwood is still unknown. The conventional induction methods somehow simulate the formation procedure of wild agarwood which is produced by physical wounding caused by heavy wind blowing, lightning striking, or insects biting [5]. However, procedures for agarwood formation by conventional methods are more time-consuming and laborious [16]. Non-conventional techniques are reported with high yielding within one-year induction but persistent accumulation of secondary metabolites inside trees are difficult [17]. Detailed comparisons on chemical constituents of artificial agarwood might also help us to know whether agarwood induction methods affect the components in resins, which will aidthe improvement of artificial induction techniques.

Chemical constituents in agarwood have been intensively studied by multiple analysis methods in recent years [1]. Main classes of aromatic constituents in agarwood are 2-(2-phenylethyl)chromones (PECs) [18]. Research conducted mainly through LC-MS/MS and NMR analysis showed chromones are grouped into four types by their backbone structures: tetrahydro-2-(2-phenylethyl)chromones (THPECs), epoxy-(2-phenylethyl)chromones (EPECs), diepoxy-(2-phenylethyl)chromones (DEPECs), and PECs [11]. Relative contents of chromones in agarwood were reported to change during agarwood formation [7]. Some reports suggested certain 2-(2-phenylethyl)chromones accumulated in high-quality agarwood [19-21]. Other reports mainly through GC-MS or NMR provide evidences for specific sesquiterpenes in agarwood of higher prices in the market $[22,23]$. Accumulative studies showed both chromones and sesquiterpenes are crucial for the medical and fragrance usages $[18,24,25]$. Therefore, chemical profiles of both chromones and sesquiterpenes are necessary for qualification of agarwood. In order to depict chemical profiles in artificial agarwood, LC-MS/MS and GC-MS combined with metabolomics were applied to compare the chemical characters of artificial agarwood in this study.

Agarwood samples separately induced by two conventional methods and two non-conventional methods were used in this study. Chemical constituents were analyzed by GC-MS and LC-MS/MS for the purpose of revealing differencs of chemical profiles in agarwood induced by different techniques. The detected compounds by GC-MS were identified by searching NIST14 or matching with reference compounds. Qualitative analysis of 2-(2-phenylethyl)chromones were conducted by UPLC-MS/MS based on previous reports. All raw data from GC-MS and LC-MS were preprocessed and further used for multivariated analysis as reported in metabolomics studies. The results strongly suggested induction methods affect chemical profiles in artifical agarwood.

\section{Results}

\subsection{Morphological Observations of Agarwoods Induced by Different Techniques}

Agarwood formation were the reactions to hurts. Resin were produced and accumulated around the wound sites. As the locations and areas of wounding sites agarwood are different in different artificial techniques, the appearances of induced agarwood varied (Figure 1 and Table 1). Wounding by an axe was tried on the trunk of trees. Agarwood covered the whole transection and are usually flakes with dark dot resins on one side (Figure 1A). The burning-chisel induced agarwood are chops with resins around the holes' wound (Figure 1B). Non-conventional inducing methods initiated the accumulation of resin inside the tree stems along the trunks or branches. Trees were chopped and white wood were carefully removed from the resinous tissues. Agarwood were regular thin chips covered with resin strips as shown in Figure 1C,D. 

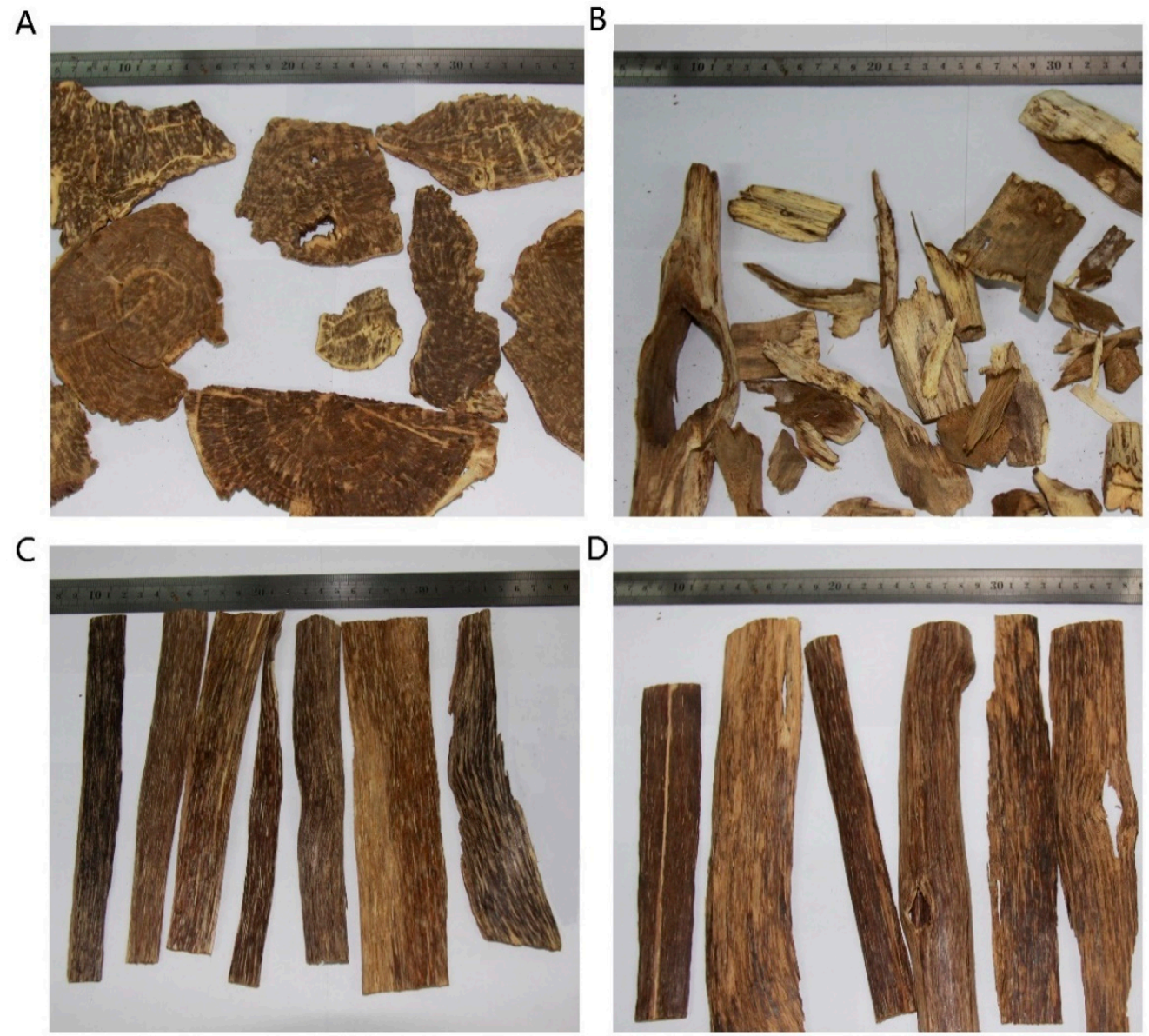

Figure 1. Morphological observations of artificial agarwood by different induction methods. (A) Wounding by axe. (B) Burning-chisel-drilling. (C) Biological inocula. (D) Chemical inducers.

Although morphology sometimes affect the commercial prices of agarwood used in sculptures or artifacts, resin contents which is scaled as ethanol extract contents are the crucial criteria in agarwood qualification system in most agarwood producing and consuming regions [26]. Resin contents partly depend on the extent of whitewood removal, especially for the artificial agarwood whose morphology is regular. Chemical constituents including chromones and sesquiterpenes in ethanol extract are contributors to the pleasant odor and medical effects of agarwood. Thus, the chemical constituents in ethanol extracts should be carefully analyzed to assess their qualities. 
Table 1. Tentative identification of chemical constituents in artificial agarwood.

\begin{tabular}{|c|c|c|c|c|c|c|c|c|}
\hline No & RT & Similarity & RIE & RIL & Compound Name & MW & Formula & Types \\
\hline 1 & 2.317 & 88 & 990 & 982 & Benzaldehyde & 106 & $\mathrm{C}_{7} \mathrm{H}_{6} \mathrm{O}$ & $\begin{array}{l}\text { Aromatic } \\
\text { compound }\end{array}$ \\
\hline 2 & 4.542 & 88 & 1156 & 1160 & Benzocyclohexane & 106 & $\mathrm{C}_{10} \mathrm{H}_{12}$ & $\begin{array}{l}\text { Aromatic } \\
\text { compound }\end{array}$ \\
\hline 3 & 6.275 & 90 & 1230 & 1228 & benzylacetone & 148 & $\mathrm{C}_{10} \mathrm{H}_{20} \mathrm{O}$ & $\begin{array}{l}\text { Aromatic } \\
\text { compound }\end{array}$ \\
\hline 4 & 19.336 & 84 & 1460 & 1454 & 7-epi-trans-seqsquisablinene hydrate & 222 & $\mathrm{C}_{15} \mathrm{H}_{26} \mathrm{O}$ & Sesquiterpenoid \\
\hline 5 & 21.000 & 93 & 1594 & l & Epi-gamma-Eudesmol & 222 & $\mathrm{C}_{15} \mathrm{H}_{26} \mathrm{O}$ & $\begin{array}{l}\text { Eudesmane-type } \\
\text { Sesquiterpenoid }\end{array}$ \\
\hline 6 & 21.912 & 95 & 1612 & 1598 & Agarospirol & 222 & $\mathrm{C}_{15} \mathrm{H}_{26} \mathrm{O}$ & $\begin{array}{l}\text { Agarospirane-type } \\
\text { Sesquiterpenoid }\end{array}$ \\
\hline 7 & 22.051 & 80 & 1615 & 1598 & Hinesol & 222 & $\mathrm{C}_{15} \mathrm{H}_{26} \mathrm{O}$ & $\begin{array}{l}\text { Guaiane-type } \\
\text { Sesquiterpenoid }\end{array}$ \\
\hline 8 & 22.273 & 82 & 1619 & 1614 & Guaiol & 222 & $\mathrm{C}_{15} \mathrm{H}_{26} \mathrm{O}$ & $\begin{array}{l}\text { Guaiane-type } \\
\text { Sesquiterpenoid }\end{array}$ \\
\hline 9 & 22.492 & 86 & 1624 & l & Tran-guaienol & 222 & $\mathrm{C}_{15} \mathrm{H}_{26} \mathrm{O}$ & $\begin{array}{l}\text { Guaiane-type } \\
\text { Sesquiterpenoid }\end{array}$ \\
\hline 10 & 22.701 & 91 & 1628 & 1614 & Guaiol & 222 & $\mathrm{C}_{15} \mathrm{H}_{26} \mathrm{O}$ & $\begin{array}{l}\text { Guaiane-type } \\
\text { Sesquiterpenoid }\end{array}$ \\
\hline $\begin{array}{l}11 \\
12\end{array}$ & $\begin{array}{l}23.334 \\
23.983\end{array}$ & $\begin{array}{l}78 \\
75\end{array}$ & $\begin{array}{l}1641 \\
1653\end{array}$ & $\begin{array}{l}1635 \\
1638\end{array}$ & $\begin{array}{l}\text { Isolongifolol } \\
\text { Zingerone }\end{array}$ & $\begin{array}{l}222 \\
194\end{array}$ & $\begin{array}{l}\mathrm{C}_{15} \mathrm{H}_{26} \mathrm{O} \\
\mathrm{C}_{11} \mathrm{H}_{14} \mathrm{O}_{3}\end{array}$ & $\begin{array}{l}\text { Sesquiterpenoid } \\
\text { Other }\end{array}$ \\
\hline 13 & 24.202 & 75 & 1658 & 1645 & 6-isopropenyl-4,8a-dimethyl-4a,5,6,7,8,8a-heahydro- $1 H$-naphthalenone & 218 & $\mathrm{C}_{15} \mathrm{H}_{22} \mathrm{O}$ & $\begin{array}{l}\text { Eudesmane-type } \\
\text { sesquiterpenoid }\end{array}$ \\
\hline 14 & 24.616 & 77 & 1669 & 1645 & (5s,7s,10s)-selina-3,11-dien-9-one & 218 & $\mathrm{C}_{15} \mathrm{H}_{22} \mathrm{O}$ & $\begin{array}{l}\text { Eudesmane-type } \\
\text { sesquiterpenoid }\end{array}$ \\
\hline 15 & 26.158 & 80 & 1698 & 1681 & 2-(4a,8-Dimethyl-1,2,3,4a,5,6,7-octahydro-naphthalenenol & 220 & $\mathrm{C}_{15} \mathrm{H}_{24} \mathrm{O}$ & $\begin{array}{l}\text { Eudesmane-type } \\
\text { sesquiterpenoid }\end{array}$ \\
\hline 16 & 26.541 & 80 & 1709 & 1645 & $(5 s, 7 s, 9 s, 10 s)-(+)$-selina-3,11-dein-9-ol & 222 & $\mathrm{C}_{15} \mathrm{H}_{26} \mathrm{O}$ & $\begin{array}{l}\text { Guaiane-type } \\
\text { Sesquiterpenoid }\end{array}$ \\
\hline 17 & 27.626 & 80 & 1719 & 1651 & Alpha endesm-11-en-1-alpha-ol & 222 & $\mathrm{C}_{15} \mathrm{H}_{26} \mathrm{O}$ & $\begin{array}{l}\text { Eudesmane-type } \\
\text { sesquiterpenoid }\end{array}$ \\
\hline 18 & 27.939 & 81 & 1743 & / & Aromadendrane-4,10-diol & 218 & $\mathrm{C}_{15} \mathrm{H}_{26} \mathrm{O}_{2}$ & Sesquiterpenoid \\
\hline 19 & 29.681 & 88 & 1761 & / & cryptomeridiol & 240 & $\mathrm{C}_{15} \mathrm{H}_{28} \mathrm{O}_{2}$ & Sesquiterpenoid \\
\hline 20 & 30.503 & 80 & 1771 & l & 2,6-Dimethyl-6-(4methyl-3-pentenyl)-2-cyclohexene-1-carboxaledehyde & 220 & $\mathrm{C}_{15} \mathrm{H}_{24} \mathrm{O}$ & $\begin{array}{l}\text { Aromatic } \\
\text { compound }\end{array}$ \\
\hline 21 & 31.181 & 80 & 1790 & l & Kessane & 220 & $\mathrm{C}_{15} \mathrm{H}_{24} \mathrm{O}$ & sesquiterpenoid \\
\hline 22 & 31.225 & 80 & 1800 & l & naphthalenone & 218 & $\mathrm{C}_{15} \mathrm{H}_{24} \mathrm{O}$ & $\begin{array}{l}\text { Eudesmane-type } \\
\text { sesquiterpenoid }\end{array}$ \\
\hline 22 & 31.499 & 75 & 1804 & / & Kessanyl acetate & 280 & $\mathrm{C}_{17} \mathrm{H}_{28} \mathrm{O}_{3}$ & sesquiterpenoid \\
\hline 23 & 31.913 & 76 & 1811 & i & Curcumenol & 234 & $\mathrm{C}_{15} \mathrm{H}_{22} \mathrm{O}_{2}$ & sesquiterpenoid \\
\hline 24 & 36.743 & 78 & 1892 & i & Benzocycloheptenol & 222 & $\mathrm{C}_{15} \mathrm{H}_{26} \mathrm{O}$ & sesquiterpenoid \\
\hline 25 & 38.025 & 75 & 1913 & 1904 & 6-[1-(Hydroxymethyl)vinyl]-4,8a-dimethyl-1,2-4a-5,6,7,8a-octahydro-2-naphthalenol & 236 & $\mathrm{C}_{15} \mathrm{H}_{24} \mathrm{O}_{2}$ & sesquiterpenoid \\
\hline 26 & 38.522 & 80 & 1921 & 1916 & naphthalenone & 234 & $\mathrm{C}_{15} \mathrm{H}_{22} \mathrm{O}_{2}$ & sesquiterpenoid \\
\hline 27 & 39.641 & 80 & 1938 & l & phenanthrenone & 250 & $\mathrm{C}_{15} \mathrm{H}_{22} \mathrm{O}_{3}$ & other \\
\hline 28 & 42.840 & 82 & 1992 & 1 & Isopimaral & 286 & $\mathrm{C}_{20} \mathrm{H}_{30} \mathrm{O}$ & diterpenes \\
\hline
\end{tabular}


Table 1. Cont.

\begin{tabular}{|c|c|c|c|c|c|c|c|c|}
\hline No & RT & Similarity & RIE & RIL & Compound Name & MW & Formula & Types \\
\hline 29 & 56.821 & 96 & 2272 & 1 & 2-(2-phenethyl)chromone & 250 & $\mathrm{C}_{17} \mathrm{H}_{14} \mathrm{O}_{2}$ & chromones \\
\hline 30 & 59.365 & 1 & 2356 & / & PEC: A ring: $1 \mathrm{OH}$ & 266 & $\mathrm{C}_{17} \mathrm{H}_{14} \mathrm{O}_{3}$ & chromones \\
\hline 31 & 62.061 & 1 & 2389 & / & PEC: A ring: $1 \mathrm{OH}$ & 266 & $\mathrm{C}_{17} \mathrm{H}_{14} \mathrm{O}_{3}$ & chromones \\
\hline 32 & 64.721 & 1 & 2458 & / & 6,8-Dihydroxy-2-(2-phenylethyl)chromone & 282 & $\mathrm{C}_{17} \mathrm{H}_{14} \mathrm{O}_{4}$ & chromones \\
\hline 33 & 64.817 & 1 & 2463 & / & PEC: A ring:1OH & 266 & $\mathrm{C}_{17} \mathrm{H}_{14} \mathrm{O}_{3}$ & chromones \\
\hline 34 & 64.882 & i & 2470 & / & PEC: $B$ ring: $1 \mathrm{OH}, 1 \mathrm{OCH}_{3}$ & 296 & $\mathrm{C}_{18} \mathrm{H}_{16} \mathrm{O}_{4}$ & chromones \\
\hline 35 & 67.430 & i & 2532 & / & PEC: A ring: $1 \mathrm{OCH}_{3}$ & 280 & $\mathrm{C}_{18} \mathrm{H}_{16} \mathrm{O}_{3}$ & chromones \\
\hline 36 & 67.533 & 94 & 2539 & / & 2-(4-Methoxyphenethyl) 4H-chromone & 280 & $\mathrm{C}_{18} \mathrm{H}_{16} \mathrm{O}_{3}$ & chromones \\
\hline 37 & 68.779 & 1 & 2569 & / & 6,8-Dihydroxy-2-(2-phenylethyl)chromone & 282 & $\mathrm{C}_{17} \mathrm{H}_{14} \mathrm{O}_{4}$ & chromones \\
\hline 38 & 69.021 & 1 & 2572 & / & 6,8-Dihydroxy-2-(2-phenylethyl)chromone & 282 & $\mathrm{C}_{17} \mathrm{H}_{14} \mathrm{O}_{4}$ & chromones \\
\hline 39 & 69.149 & 1 & 2579 & / & 6-Hydroxy-8-chloro-2-(2-phenylethyl)chromone & 300 & $\mathrm{C}_{17} \mathrm{H}_{13} \mathrm{ClO}_{3}$ & chromones \\
\hline 40 & 69.350 & 1 & 2583 & / & PEC: A ring: $1 \mathrm{OH}, 1 \mathrm{OCH}_{3}$ & 296 & $\mathrm{C}_{18} \mathrm{H}_{16} \mathrm{O}_{4}$ & chromones \\
\hline 41 & 71.133 & & 2633 & & Agaretetrol & 318 & $\mathrm{C}_{17} \mathrm{H}_{18} \mathrm{O}_{6}$ & chromones \\
\hline 42 & 71.355 & 1 & 2639 & 1 & PEC: A ring: $1 \mathrm{OH}$, & 266 & $\mathrm{C}_{17} \mathrm{H}_{14} \mathrm{O}_{3}$ & chromones \\
\hline 43 & 72.773 & 1 & 2678 & / & EPEC: $B$ ring: $1 \mathrm{OH}$ & 284 & $\mathrm{C}_{17} \mathrm{H}_{16} \mathrm{O}_{4}$ & chromones \\
\hline 44 & 72.873 & 1 & 2683 & / & PEC: $B$ ring: $1 \mathrm{OH}, 1 \mathrm{OCH}_{3}$ & 296 & $\mathrm{C}_{18} \mathrm{H}_{16} \mathrm{O}_{4}$ & chromones \\
\hline 45 & 74.124 & 1 & 2716 & / & PEC: A ring: $1 \mathrm{OH}, 1 \mathrm{OCH}_{3}, \mathrm{~B}$ ring: $1 \mathrm{OCH}_{3}$ & 326 & $\mathrm{C}_{19} \mathrm{H}_{18} \mathrm{O}_{5}$ & chromones \\
\hline 46 & 74.852 & 1 & 2734 & / & 5,8-Dihydroxy-2-[2-(4-methoxyphenyl)ethyl]chromone & 312 & $\mathrm{C}_{18} \mathrm{H}_{16} \mathrm{O}_{5}$ & chromones \\
\hline 47 & 75.450 & 1 & 2751 & / & EPEC: A ring: $1 \mathrm{OH}, 1 \mathrm{OCH}_{3}, 1 \mathrm{epo}$ & 314 & $\mathrm{C}_{18} \mathrm{H}_{18} \mathrm{O}_{5}$ & chromones \\
\hline 48 & 76.361 & 1 & 2780 & i & EPEC: A ring: $2 \mathrm{OH}, 1$ epo & 300 & $\mathrm{C}_{17} \mathrm{H}_{16} \mathrm{O}_{5}$ & chromones \\
\hline 49 & 77.476 & 1 & 2813 & / & PEC:B ring: $2 \mathrm{OCH}_{3}$ & 310 & $\mathrm{C}_{19} \mathrm{H}_{18} \mathrm{O}_{4}$ & chromones \\
\hline 50 & 77.678 & 1 & 2819 & i & PEC: A ring: $2 \mathrm{OH}, \mathrm{B}$ ring: $1 \mathrm{OCH}_{3}$ & 312 & $\mathrm{C}_{18} \mathrm{H}_{16} \mathrm{O}_{5}$ & chromones \\
\hline 51 & 79.350 & 1 & 2863 & / & PEC: A ring: $1 \mathrm{OH}, 1 \mathrm{OCH}_{3}$ & 296 & $\mathrm{C}_{18} \mathrm{H}_{16} \mathrm{O}_{4}$ & chromones \\
\hline 52 & 80.050 & 1 & 2890 & / & Stigmasterol & 412 & $\mathrm{C}_{29} \mathrm{H}_{4} 8 \mathrm{O}$ & stigmasterol \\
\hline 53 & 80.208 & 1 & 2901 & / & THPEC: A ring: $4 \mathrm{OH} \mathrm{B}$ ring $1 \mathrm{OCH}_{3}$ & 348 & $\mathrm{C}_{18} \mathrm{H}_{20} \mathrm{O}_{7}$ & chromones \\
\hline 54 & 80.616 & 1 & 2907 & / & PEC: A ring: $1 \mathrm{OH}, \mathrm{B}$ ring: $1 \mathrm{OCH} 3$ & 296 & $\mathrm{C}_{18} \mathrm{H}_{16} \mathrm{O}_{4}$ & chromones \\
\hline 55 & 81.142 & 1 & 2923 & / & PEC: A ring: $2 \mathrm{OH}, \mathrm{B}$ ring: $1 \mathrm{OCH} 3,1 \mathrm{OH}$ & 328 & $\mathrm{C}_{18} \mathrm{H}_{16} \mathrm{O}_{6}$ & chromones \\
\hline 56 & 81.676 & 1 & 2939 & / & PEC: A ring: $1 \mathrm{OCH}_{3}, \mathrm{~B}$ ring: $1 \mathrm{OCH}_{3}, 1 \mathrm{OH}$ & 326 & $\mathrm{C}_{19} \mathrm{H}_{18} \mathrm{O}_{5}$ & chromones \\
\hline 57 & 83.152 & 1 & 2985 & 1 & PEC: A ring: $1 \mathrm{OCH}_{3}, 1 \mathrm{OH} ; \mathrm{B}$ ring: $1 \mathrm{OCH}_{3}, 1 \mathrm{OH}$ & 342 & $\mathrm{C}_{19} \mathrm{H}_{18} \mathrm{O}_{6}$ & chromones \\
\hline 58 & 83.484 & i & 2995 & / & PEC: A ring: $1 \mathrm{OCH}_{3}, \mathrm{~B}$ ring: $1 \mathrm{OCH}_{3}, 1 \mathrm{OH}$ & 326 & $\mathrm{C}_{19} \mathrm{H}_{18} \mathrm{O}_{5}$ & chromones \\
\hline 59 & 83.821 & 1 & 3006 & 1 & PEC: A ring: $2 \mathrm{OH}, \mathrm{B}$ ring: $1 \mathrm{OCH}_{3}, 1 \mathrm{OH}$ & 328 & $\mathrm{C}_{18} \mathrm{H}_{16} \mathrm{O}_{6}$ & chromones \\
\hline 60 & 84.961 & / & 3042 & & PEC: A ring: $1 \mathrm{OCH}_{3}, 2 \mathrm{OH}$; $\mathrm{B}$ ring: $1 \mathrm{OCH}_{3}$, & 342 & $\mathrm{C}_{19} \mathrm{H}_{18} \mathrm{O}_{6}$ & chromones \\
\hline 61 & 85.498 & 1 & 3059 & & PEC: A ring: $1 \mathrm{OH}, \mathrm{B}$ ring: $1 \mathrm{OCH}_{3}, 1 \mathrm{OH}$ & 312 & $\mathrm{C}_{18} \mathrm{H}_{16} \mathrm{O}_{5}$ & chromones \\
\hline 62 & 85.916 & i & 3073 & / & PECs: A ring: $2 \mathrm{OCH}_{3}, \mathrm{~B}: 1 \mathrm{OCH}_{3}$ & 340 & $\mathrm{C}_{20} \mathrm{H}_{20} \mathrm{O}_{5}$ & chromones \\
\hline 63 & 86.952 & 1 & 3106 & / & PEC: A ring: $1 \mathrm{OH}, \mathrm{B}$ ring: $1 \mathrm{OCH}_{3}, 1 \mathrm{OH}$ & 312 & $\mathrm{C}_{18} \mathrm{H}_{16} \mathrm{O}_{5}$ & chromones \\
\hline 64 & 87.695 & 1 & 3130 & / & PEC: A ring: $1 \mathrm{OCH}_{3}, 1 \mathrm{OH} ; \mathrm{B}$ ring: $1 \mathrm{OCH} 3$ & 326 & $\mathrm{C}_{19} \mathrm{H}_{18} \mathrm{O}_{5}$ & chromones \\
\hline 65 & 88.537 & 88 & 3178 & / & stigmasterol & 412 & $\mathrm{C}_{29} \mathrm{H}_{48} \mathrm{O}$ & Steriods \\
\hline 66 & 89.950 & / & 3205 & i & PEC: A ring: $1 \mathrm{OCH}_{3}, 1 \mathrm{OH} ; \mathrm{B}$ ring: $1 \mathrm{OCH}_{3}, 1 \mathrm{OH}$ & 342 & $\mathrm{C}_{19} \mathrm{H}_{18} \mathrm{O}_{6}$ & chromones \\
\hline 67 & 90.813 & 92 & 3234 & / & gamma-Sitosterol & 414 & $\mathrm{C}_{29} \mathrm{H}_{50} \mathrm{O}$ & Steriods \\
\hline 68 & 91.042 & 1 & 3242 & / & PEC: A ring: $1 \mathrm{Cl}, 1 \mathrm{OH} ; \mathrm{B}$ ring: $1 \mathrm{OCH}_{3}, 1 \mathrm{OH}$ & 346 & $\mathrm{C}_{18} \mathrm{H}_{15} \mathrm{ClO}_{5}$ & chromones \\
\hline 69 & 91.814 & 1 & 3268 & / & PEC: A ring: $2 \mathrm{OCH}_{3} ; \mathrm{B}$ ring: $1 \mathrm{OCH}_{3}, 1 \mathrm{OH}$ & 356 & $\mathrm{C}_{20} \mathrm{H}_{20} \mathrm{O}_{6}$ & chromones \\
\hline 70 & 93.730 & 1 & 3334 & / & PEC: A ring: $1 \mathrm{OCH}_{3}, 1 \mathrm{OH} ; \mathrm{B}$ ring: $1 \mathrm{OCH}_{3}, 1 \mathrm{OH}$ & 342 & $\mathrm{C}_{19} \mathrm{H}_{18} \mathrm{O}_{6}$ & chromones \\
\hline 71 & 94.495 & 87 & 3361 & 1 & Gamma-Sitostenone & 412 & $\mathrm{C}_{29} \mathrm{H}_{48} \mathrm{O}$ & Steriods \\
\hline
\end{tabular}

"/" not determined, PEC, 2-(2-phenylethyl)chromone; THPECs, tetrahydro-2-(2-phenylethyl) chromones; EPECs, epoxy-(2-phenylethyl)chromones; diepoxy-(2-phenylethyl)chromones (DEPECs). 


\subsection{GC-MS Analysis}

As agarwood is valued for its unique and pleasant odor, GC-MS were widely used in determining the components and access the quality. To fully acquire the chemical profiles of agarwoods, the ethanol extracts were eluted and analyzed by GC-MS. The typical TIC chromatographs of agarwood by four inducing methods are shown in Figure 2. Chemicals that showed up before retention time (RT) of 57 min were mainly sesquiterpenes and aromatic compounds. PECs were detected between 57 and $87 \mathrm{~min}$ with some long chain fatty acid. Steroids showed up in the last $15 \mathrm{~min}$. Chemicals, except for some PECs, were identified by searching NIST14 with MS data and retention index. Tentatively identified chemicals with similarity over $80 \%$ and retention index within \pm 20 are listed in Table 1 . Some PECs identity was done by referring to previous reports. Proportion of sesquiterpenoid was highest in groupA. PECs made up most proportions of chemicals detected in burning-chisel and non-conventional method induced agarwood (Figure 2). However, THPECs overlapped with DPECs and EPECs in GC-MS analysis (Figure 2B).
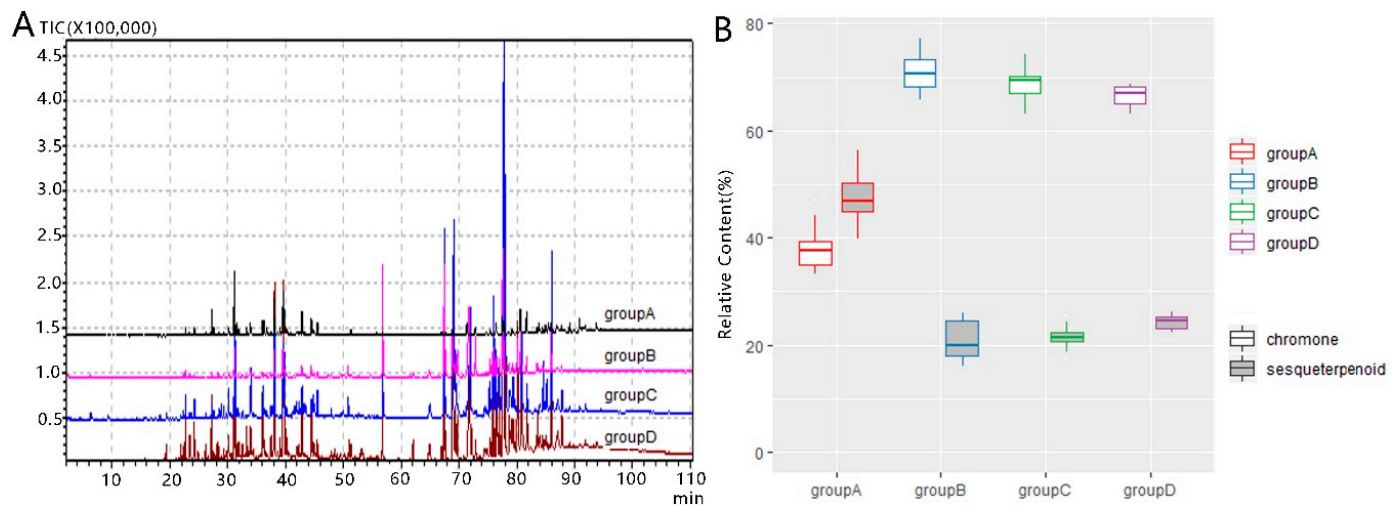

Figure 2. GC-MS analysis of artificial agarwood induce by different techniques. (A) Representative chromatographs of artificial agarwood. (B) Relative contents of main components detected by GC-MS. group A, wounding by axe; group B, burning-chisel-drilling; group C, chemical inducers; group D, biological inocula.

However, GC-MS is only suitable for volatile constituents and identification via GC-MS can only be achieved within limited library searching. Therefore, given the fact that PECs comprised a high percentage of the identified compounds, and while some nonvolatile PECs perhaps failed to be detected by GC-MS, UPLC-MS analysis was carried out to study the dynamic changes of PECs during the process of agarwood formation.

\subsection{LC-MS Analysis}

2-(2-phenylethyl) chromones are widely reported as the characteristic constituents in agarwood in regardless of their origins and induction methods. The chromone patterns of each groups were acquired by LC-MS/MS. Total ion chromatograph (TIC) obtained by scan mode showed that all artificial agarwood were qualitatively similar (Figure 3A). Basal peaks were chosen and further identified by LC-MS/MS by production ion mode. All four types of 2-(2-phenylethyl) chromones were detected in artificial agarwood and their backbone structures are shown in Figure 3B.

Agarotetrol, 2-[2-(4-methoxphenyl)ethyl] chromone and 2-(2-phenylethly) chromone were identified by comparing their retention times and MS spectra with reference compounds. Tentative identification of chromones according to MS/MS fragments and previous reports are listed in Table 1. THPEC with 4 OHs at ring A were eluted firstly before $7 \mathrm{~min}$. THPECs with less than 4 OHs, EPECs and DEPECs appear between 7 and $13 \mathrm{~min}$. Chemicals detected after $13 \mathrm{~min}$ were molecular with least polarity such as FTPECs and sesquiterpenoids (Figure 3 and Table 2). Proportions of each type of chromone in artificial agarwood samples and the most abundant chemical was shown in Figure 4. 
FTPECs comprise most part of chromones in artificial agarwood (Figure 4). THPECs is lower while EPECs and DEPECS were more abundant in group A than in other groups (Figure 4). The above results strongly suggested chromones in artificial agarwood differ between groups.

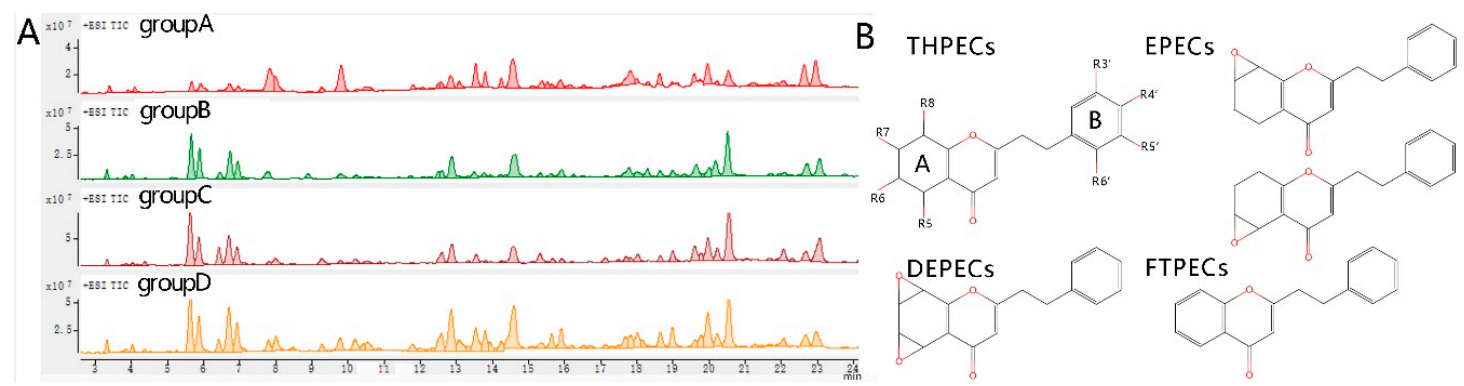

Figure 3. Total ion chromatograph (TIC) of agarwood produced by artificial induction methods (A) and the characteristic structures of four types of 2-(2-phenylethyl)chromones, (B) detected by LC-MS. group A, wounding by axe; group B, burning-chisel-drilling; group C, chemical inducers; group D, biological inocula.

Table 2. Tentative identification of 2-(2-phenylethyl) chromones (PECs) in artificial agarwood.

\begin{tabular}{|c|c|c|c|c|c|}
\hline No & RT (min) & MF & MW & Compounds & Fragment Ions \\
\hline 1 & 2.628 & $\mathrm{C}_{19} \mathrm{H}_{23} \mathrm{O}_{9}$ & 394 & THPECs: A ring: $4 \mathrm{OH}, \mathrm{B}$ ring: $1 \mathrm{OH}, 2 \mathrm{OCH}_{3}$ & $377,361,331,167$ \\
\hline 2 & 3.328 & $\mathrm{C}_{18} \mathrm{H}_{21} \mathrm{O}_{8}$ & 364 & THPECs: A ring: $4 \mathrm{OH}, \mathrm{B}$ ring: $1 \mathrm{OH}, 1 \mathrm{OCH}_{3}$ & $347,329,301,137$ \\
\hline 3 & 3.85 & $\mathrm{C}_{18} \mathrm{H}_{21} \mathrm{O}_{8}$ & 364 & THPECs: A ring: $4 \mathrm{OH}, \mathrm{B}$ ring: $1 \mathrm{OH}, 1 \mathrm{OCH}_{3}$ & $347,329,301,137$ \\
\hline 5 & 5.616 & $\mathrm{C}_{17} \mathrm{H}_{18} \mathrm{O}_{6}$ & 318 & Agarotetrol * & $301,283,255,91$ \\
\hline 6 & 5.876 & $\mathrm{C}_{18} \mathrm{H}_{20} \mathrm{O}_{7}$ & 348 & THPECs: A ring: $4 \mathrm{OH}, \mathrm{B}$ ring: $1 \mathrm{OCH}_{3}$ & $331,313,121$ \\
\hline 7 & 6.4 & $\mathrm{C}_{17} \mathrm{H}_{18} \mathrm{O} 6$ & 318 & THPECs: A ring: $4 \mathrm{OH}$ & $301,283,255,91$ \\
\hline 10 & 7.788 & $\mathrm{C}_{18} \mathrm{H}_{18} \mathrm{O}_{7}$ & 346 & EPECs: A ring:2 $\mathrm{OH}, \mathrm{B}$ ring: $1 \mathrm{OH}, 1 \mathrm{OCH}_{3}$ & $329,301,137$ \\
\hline 11 & 7.988 & $\mathrm{C}_{18} \mathrm{H}_{18} \mathrm{O}_{7}$ & 346 & EPECs: A ring:2OH, $\mathrm{B}$ ring: $1 \mathrm{OH}, 1 \mathrm{OCH}_{3}$ & $329,301,137$ \\
\hline 12 & 7.988 & $\mathrm{C}_{18} \mathrm{H}_{18} \mathrm{O}_{7}$ & 358 & DEPECs: $\mathrm{B}$ ring $1 \mathrm{OH}, 2 \mathrm{OCH}_{3}$ & 329,167 \\
\hline 13 & 8.427 & $\mathrm{C}_{18} \mathrm{H}_{18_{6}} \mathrm{O}$ & 330 & EPECs: A ring:2 $\mathrm{OH}$, $\mathrm{B}$ ring: $1 \mathrm{OCH}_{3}$ & 311,121 \\
\hline 14 & 8.992 & $\mathrm{C}_{18} \mathrm{H}_{16} \mathrm{O}_{6}$ & 328 & DEPECs: $\mathrm{B}$ ring $1 \mathrm{OH}, 1 \mathrm{OCH}_{3}$ & 137 \\
\hline 19 & 11.783 & $\mathrm{C}_{18} \mathrm{H}_{18} \mathrm{O}$ & 328 & DEPECs: $\mathrm{B}$ ring: $1 \mathrm{OCH}_{3}, 1 \mathrm{OH}$ & 301,137 \\
\hline 20 & 12.505 & $\mathrm{C}_{18} \mathrm{H}_{20} \mathrm{ClO}_{6}$ & 366 & THPECs: A ring: $3 \mathrm{OH}, 1 \mathrm{Cl} \mathrm{OCH} 3, \mathrm{~B}$ ring: $1 \mathrm{OCH}_{3}$ & 349,121 \\
\hline 21 & 12.583 & $\mathrm{C}_{17} \mathrm{H}_{18} \mathrm{ClO}_{5}$ & 336 & THPECs: A ring: $1 \mathrm{Cl}, 3 \mathrm{OH}$ & $319,301,283,273,91$ \\
\hline 22 & 12.849 & $\mathrm{C}_{19} \mathrm{H}_{22} \mathrm{O}_{5}$ & 330 & THPECs: A ring: $3 \mathrm{OH}$, B ring: $1 \mathrm{OCH}_{3}$ & 313,121 \\
\hline 23 & 13.083 & $\mathrm{C}_{19} \mathrm{H}_{16} \mathrm{O}_{6}$ & 342 & FTPECs: A ring: $1 \mathrm{OCH}_{3}, 1 \mathrm{OH} \mathrm{B}$ ring: $1 \mathrm{OCH}_{3}, 1 \mathrm{OH}$ & 237,137 \\
\hline 24 & 13.538 & $\mathrm{C}_{19} \mathrm{H}_{16} \mathrm{O}_{6}$ & 342 & FTPECs: A ring: $1 \mathrm{OCH}_{3}, 1 \mathrm{OH} \mathrm{B}$ ring: $1 \mathrm{OCH}_{3}, 1 \mathrm{OH}$ & 237,137 \\
\hline 25 & 13.805 & $\mathrm{C}_{18} \mathrm{H}_{18} \mathrm{O}$ & 312 & FTPECs: A ring:1OH, B ring: $1 \mathrm{OCH}_{3}, 1 \mathrm{OH}$ & 137 \\
\hline 26 & 14.244 & $\mathrm{C}_{18} \mathrm{H}_{16} \mathrm{O}_{5}$ & 312 & FTPECs: A ring:2OH, B ring: $1 \mathrm{OCH}_{3}$ & 121 \\
\hline 27 & 14.556 & $\mathrm{C}_{18} \mathrm{H}_{16} \mathrm{O}_{5}$ & 312 & FTPECs: A ring: $1 \mathrm{OH}, 1 \mathrm{OCH} 3 \mathrm{~B}$ ring: $1 \mathrm{OH}$ & 107 \\
\hline 28 & 15.105 & $\mathrm{C}_{19} \mathrm{H}_{18} \mathrm{O}_{5}$ & 326 & FTPECs: A ring: $2 \mathrm{OH}, \mathrm{B}$ ring: $1 \mathrm{OH}, 1 \mathrm{OCH}_{3}$ & 137 \\
\hline 29 & 15.372 & $\mathrm{C}_{20} \mathrm{H}_{20} \mathrm{O}_{6}$ & 356 & FTPECs: A ring: $\mathrm{OH}, 2 \mathrm{OCH}_{3}, \mathrm{~B}$ ring: $1 \mathrm{OCH}_{3}$ & 121, \\
\hline 30 & 15.899 & $\mathrm{C}_{20} \mathrm{H}_{20} \mathrm{O}_{6}$ & 356 & FTPECs: A ring: $2 \mathrm{OCH}_{3}$, B ring: $1 \mathrm{OH}, 1 \mathrm{OCH}_{3}$ & 137,221 \\
\hline 39 & 20.549 & $\mathrm{C}_{19} \mathrm{H}_{18} \mathrm{O}_{4}$ & 310 & FTPECs: A ring: $2 \mathrm{OCH}_{3}$ & 91 \\
\hline 40 & 21.688 & $\mathrm{C}_{18} \mathrm{H}_{16} \mathrm{O}_{3}$ & 280 & 2-[2-(4-Methoxyphenyl)ethyl] chromone * & 121 \\
\hline 41 & 22.088 & $\mathrm{C}_{17} \mathrm{H}_{14} \mathrm{O}_{2}$ & 250 & 2-(2-phenyethyl) chromone * & 91 \\
\hline 42 & 20.232 & $\mathrm{C}_{20} \mathrm{H}_{20} \mathrm{O}_{5}$ & 340 & FTPECs: A ring: $2 \mathrm{OCH}_{3}, \mathrm{~B}$ ring: $1 \mathrm{OCH}_{3}$ & 121 \\
\hline 43 & 20.549 & $\mathrm{C}_{19} \mathrm{H}_{18} \mathrm{O}_{4}$ & 310 & FTPECs: A ring: $2 \mathrm{OCH}_{3}$ & 91 \\
\hline
\end{tabular}




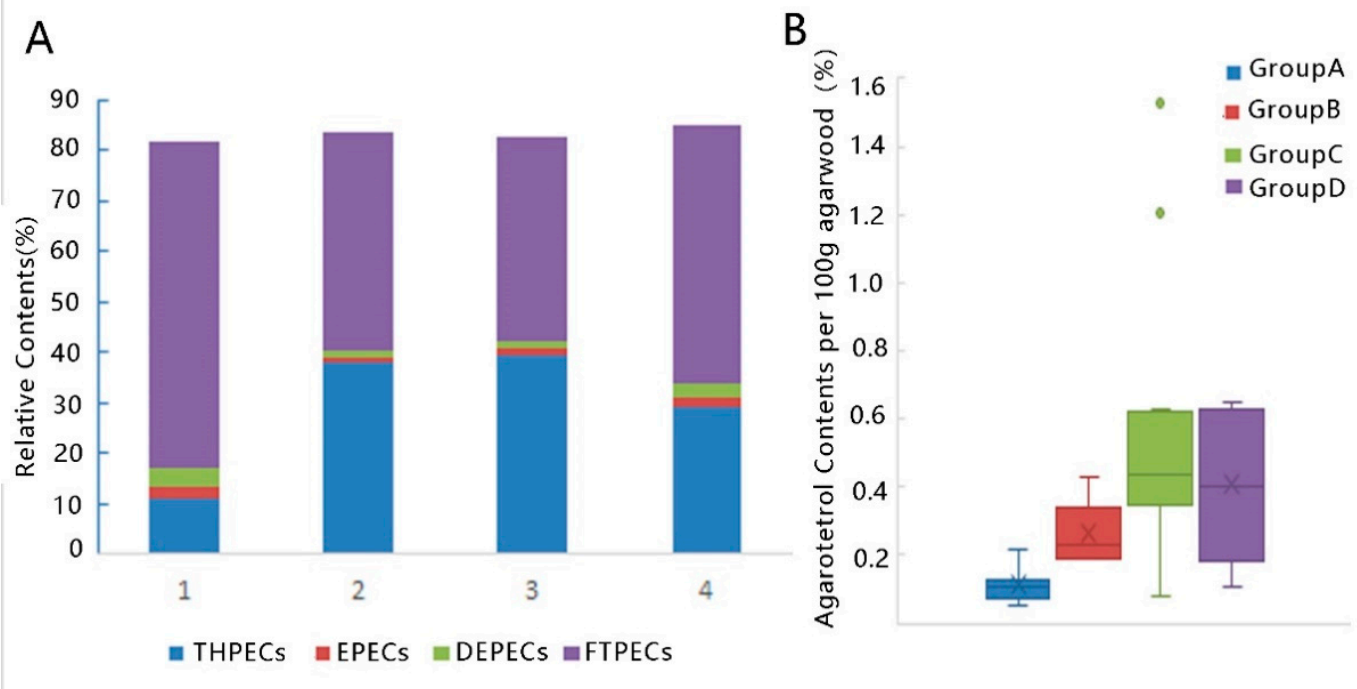

Figure 4. Contents of PECs in artificial agarwood groups. (A) Percentiles of four types of PECs in four artificial agarwood group. (B) Contents of agarotetrol $(\mathrm{g} / 100 \mathrm{~g})$ in artificial agarwood groups. group A, wounding by axe; group B, burning-chisel-drilling; group C, chemical inducers; group D, biological inocula.

\subsection{Multivariate Analysis}

As the above results show, chemical constituents in artificial agarwood are complex. To further investigate the differences within groups, all MS data were further processed for multivariate analysis. PCA respectively based on GC-MS and LC-MS showed similar grouping tendency. Most samples of group A were allocated together while samples for other groups mixed up (Figure 5A,B). Samples from group B (blue dots) scattered which suggested great variety both in sesquiterpenes and PECs.
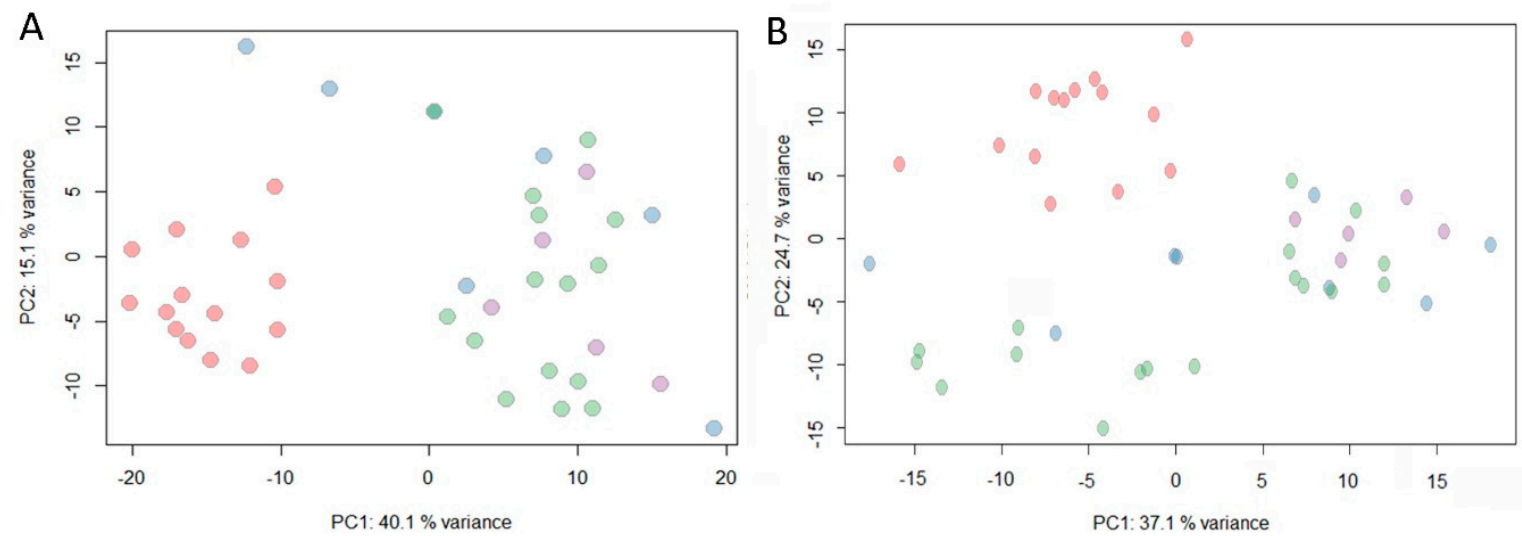

Figure 5. PCA based on (A) GC-MS and (B) LC-MS. Red, group A; blue, group B; green, group C; purple, group D.

To further identify the molecules contributing to the classification of artificial agarwood, LC-MS data were used for features selection for each group. 26 chemicals were confirmed important for artificial agarwood grouping. Random Forest based on the intensity of above 26 chemicals showed that 9 with VIP $>1.5$ (Figure 6B). Those molecules were further identified by LC-MS/MS and the possible formula were listed in Table 3 

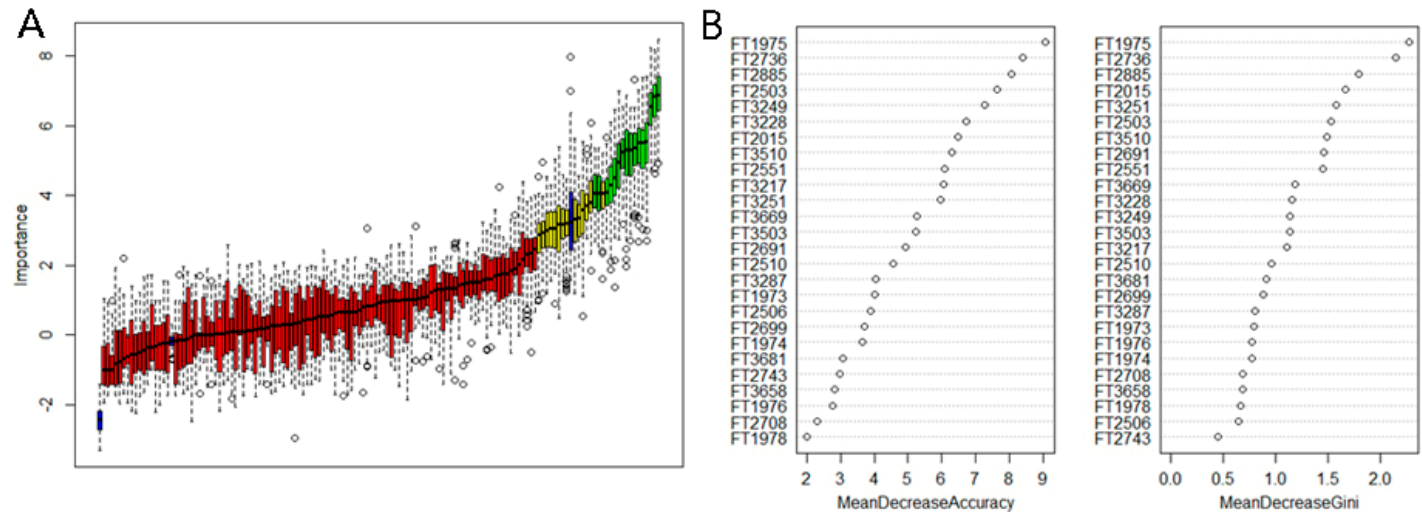

Figure 6. Random forest model based on LC-MS data (A) Feature selection based on variables used in PCA. Green, attributes confirmed important; yellow, tentative attributes; red, attributes confirmed unimportant. (B) VIP of factors contributing to artificial agarwood grouping in Random Forest model based on attributes selected in A.

Table 3. Tentatively identified chemicals contributing to artificial agarwood grouping by LC-MS/MS.

\begin{tabular}{ccccc}
\hline No & RT & MW & Type & $\begin{array}{c}\text { Molecular } \\
\text { Formula }\end{array}$ \\
\hline FT1975 & 14.6 & 334 & THPECs: A ring: $4 \mathrm{OH}, \mathrm{B}$ ring: $1 \mathrm{OH}$ & $\mathrm{C}_{17} \mathrm{H}_{18} \mathrm{O}_{7}$ \\
FT2736 & 17.916 & 326 & FTPECs: A ring: $2 \mathrm{OCH}, \mathrm{B}$ ring: $1 \mathrm{OH}$ & $\mathrm{C}_{19} \mathrm{H}_{18} \mathrm{O}_{5}$ \\
FT2885 & 17.633 & 266 & FTPECs: B ring: $1 \mathrm{OH}$ & $\mathrm{C}_{17} \mathrm{H}_{14} \mathrm{O}_{3}$ \\
FT2015 & 14.7 & 304 & Unknown & $\mathrm{C}_{20} \mathrm{H}_{32} \mathrm{O}_{2}$ \\
FT3251 & 19.333 & 300 & FTPECs: A ring: $1 \mathrm{OH}, 1 \mathrm{Cl}$ & $\mathrm{Cl}_{7} \mathrm{H}_{13} \mathrm{ClO}_{3}$ \\
FT2503 & 16.033 & 282 & Sesquiterpenoid & $\mathrm{C}_{16} \mathrm{H}_{26} \mathrm{O}_{4}$ \\
FT3510 & 19.85 & 218 & Sesquiterpenoid & $\mathrm{C}_{15} \mathrm{H}_{22} \mathrm{O}$ \\
FT2691 & 16.567 & 296 & FTPECs: A ring: $1 \mathrm{OH}, 1 \mathrm{OCH}_{3}, \mathrm{~B}$ ring: $1 \mathrm{OCH}_{3}$ & $\mathrm{C}_{18} \mathrm{H}_{16} \mathrm{O}_{4}$ \\
FT2551 & 17.85 & 326 & FTPECs: A ring: $2 \mathrm{OCH}_{3}, \mathrm{~B}$ ring: $1 \mathrm{OH}$ & $\mathrm{C}_{19} \mathrm{H}_{18} \mathrm{O}_{5}$ \\
\hline
\end{tabular}

THEPECs, Tetrahydro-2-(2-phenylethyl)-chromones; FTPECs, 2-(2-phenylethyl)-chromones.

Nearly all chemicals contributing to artificial agarwood classification came out at RT 14-19 min, which are most tentatively identified as FTPECs or sesquiterpenes with less polarity (Table 3).

\section{Discussion}

In this study, morphology and chemical profile of artificial agarwood by four induction methods were investigated. Artificial agarwood from different induction methods vary and results acquired from multivariate analysis strongly suggested that inducing methods affect the chemical constituents of artificial agarwood. There are over 200 chemicals found in agarwood and the number is increasingly growing. In this study, GC-MS tentatively identified 71 and LC-MS/MS tentatively identified 43 chemicals in artificial agarwood (Tables 1 and 2). All samples had similar chemicals in the ethanol extracts while the relative contents differed (Figures 2 and 4).

Unlike PECs in agarwood, although numerous sesquiterpenoids were tentatively identified in GC-MS (Table 1), no characteristic chemicals were found even in group A. One reason for relative low proportion of sesquiterpenoids in all samples is that the variety of sesquiterpenoid skeletons made them difficult to be identified without standard chemicals. Samples from group A were obviously different from other samples for its higher contents of sesquiterpenoids and lower contents of THEPCs (Figures 2 and 4). One explanation for the relative lower proportions of sesquiterpenoids might be the shorter formation time in group B-D compared with group A. Sesquiterpenoids were reported be produced later than PECs [15]. THPECs are reported appear early during agarwood formation and evolved into FPECs as time goes on [11]. The formation time of samples in group A is usually over 18 months. Many samples in group B-D are about 9-16 months if known. Time length of agarwood formation 
might be a crucial contributor for the differences between group A and other groups. However, other factors might also contribute to the chemical composition differences. As reports on mechanisms of agarwood formation suggest differences reside between the wounding by an axe and chemical inducer method through transcriptome and microtome analysis $[2,27,28]$. Whether those divergences accounts for the chemical variety of artificial agarwood should be an interesting question to be resolved.

Previous studies focused on agarwood qualifications proposed certain sesquiterpenes and PECs were accumulated in high-quality agarwood. In this study, agarwood in group A contained more sesquiterpenes and PECs than other groups (Figures 2 and 4), which suggested that agarwood in group A might be superior than other groups. Although many of the chemicals identified in this study can also been found in other plants, the complexity and variety of chemicals in agarwood confer its irreplaceable odor and pharmaceutical effects. Results from random forest point out several chemicals strongly correlated with samples groupings (Figure 6). It is still hard to conclude that those molecules are quality determinants. Further studies combining both chemicals analysis and medical effects or fragrant assays might help to identify the qualification markers.

Agarwood formation is believed to be the co-production of microbe and parenchyma cell at the injured sites as reported in recent research. Micro-environment of agarwood formation in conventional methods induced agarwood and the non-conventional ones differs, which can be observed by the morphology of sample in Figure 1A-D. Samples from group A were exposed to air and sunlight in whole inducing procedure while samples in group $C-D$ were in dark and anaerobic niche until harvest. Agarwood in group B was produced in both niches; as shown in Figure 1B, holes were opened to the outside and the part near the hole were covered inside trunks. Group allocations by PCA on chemical profiles also reflect the tendency (Figure 5). Group B is closer to group A than groups $C$ and D (Figures 5 and 6). Samples in group $C$ and group D were similar in morphology and chemical profiles (Figures 1 and 6). The results strongly suggested that inducing methods affect the chemicals in cultivated agarwood. Studies focused on the microtomic and biological activities in agarwood formation under different niches might shed more light on the complex mechanisms of agarwood formation.

Agarwood is precious due to the rare formation under natural environment. Various artificial inducing techniques have been developed. In this study, cultivated agarwood induced by four methods were analyzed and compared. Types of components in the ethanol extracts are similar while differing in proportions, which strongly suggest that inducing method affect chemicals in resins of agarwood. The results are helpful for study of mechanism behind agarwood formation. Agarwood induced by wounding of axes contains more sesquiterpenes and FPECs compared with other groups. The results provide a comprehensive assessment of agarwood induced by four popular artificial methods which will help to evaluate artificial agarwood quality.

\section{Materials and Methods}

\subsection{Agarwood Materials and Reagents}

All agarwood were from plantations in Guangdong, Guangxi, Yunnan, and Hainan provinces in South China. In total, 48 artificial agarwood induced by wounding using an axe (14), burning-chiseldrilling (9), chemical inducers (17) and biological inoculation (8) were investigated. Detailed sample information was listed in Table 4. The alkaline standard (D-2887 Calibration Solution, J\&K Scientific, Beijing, China) was used in GC-MS. LC-MS grade acetonitrile (Merck, Darmstadt, Germany), formic acid (TCI, Japan, Shanghai). Ultra-high purity water prepared by filtration using a Milli-Q system (Millipore, Milford, MA, USA) were used as solvents for LC-MS. Lab grade ethanol and ethyl acetate were purchased from Sigma-Aldrich (St. Louis, MO, USA). Agaroterol (111980-201602) and reference agarwood (121222-201203) were purchased from National Institutes for Food and Drug Control of China (Beijing, China). 2-(2-phenylethyl)chromone and 2-[2-(4-Methoxyphenyl)ethyl] chromone were generously gifted by Dr. Dai Haofu. Before the study, all samples were analyzed according the 
instructions of monography "agarwood" in Chinese Pharmacopoeia (2015 edition) to make sure the fake or adulterated samples were excluded from the following study [29].

Table 4. Sample information.

\begin{tabular}{|c|c|c|c|c|c|c|c|c|}
\hline No & Group & Origin & No & Group & Origin & No & Group & Origin \\
\hline $\mathrm{S} 1$ & A & $\begin{array}{l}\text { Zhongshan, } \\
\text { Guangdong }\end{array}$ & S17 & B & Haikou, Hainan & S33 & C & Haikou, Hainan \\
\hline S2 & A & $\begin{array}{l}\text { Zhongshan, } \\
\text { Guangdong }\end{array}$ & S18 & B & $\begin{array}{l}\text { Dongguan, } \\
\text { Guangdong }\end{array}$ & S34 & C & Haikou, Hainan \\
\hline S3 & A & $\begin{array}{l}\text { Zhongshan, } \\
\text { Guangdong }\end{array}$ & S19 & B & $\begin{array}{l}\text { Dongguan, } \\
\text { Guangdong }\end{array}$ & S35 & C & Haikou, Hainan \\
\hline $\mathrm{S} 4$ & A & $\begin{array}{l}\text { Dongguan, } \\
\text { Guangdong }\end{array}$ & S20 & B & $\begin{array}{l}\text { Dongguan, } \\
\text { Guangdong }\end{array}$ & S36 & C & Haikou, Hainan \\
\hline S5 & A & $\begin{array}{l}\text { Dongguan, } \\
\text { Guangdong }\end{array}$ & $\mathrm{S} 21$ & B & Haikou, Hainan & S37 & C & Haikou, Hainan \\
\hline S6 & A & $\begin{array}{l}\text { Dongguan, } \\
\text { Guangdong }\end{array}$ & $\mathrm{S} 22$ & B & Haikou, Hainan & S38 & C & Haikou, Hainan \\
\hline S7 & A & $\begin{array}{l}\text { Dongguan, } \\
\text { Guangdong }\end{array}$ & $\mathrm{S} 23$ & B & Haikou, Hainan & S39 & C & Haikou, Hainan \\
\hline S8 & A & $\begin{array}{l}\text { Dongguan } \\
\text { Guangdong }\end{array}$ & S24 & C & Yulin, Guangxi & $\mathrm{S} 40$ & C & $\begin{array}{l}\text { Zhongshan, } \\
\text { Guangdong }\end{array}$ \\
\hline S9 & $\mathrm{A}$ & $\begin{array}{l}\text { Dongguan, } \\
\text { Guangdong }\end{array}$ & S25 & C & $\begin{array}{l}\text { Zhongshan, } \\
\text { Guangdong }\end{array}$ & S41 & $\mathrm{D}$ & Puer, Yunan \\
\hline S10 & A & $\begin{array}{l}\text { Dongguan, } \\
\text { Guangdong }\end{array}$ & S26 & C & $\begin{array}{l}\text { Zhongshan, } \\
\text { Guangdong }\end{array}$ & $\mathrm{S} 42$ & $\mathrm{D}$ & Puer, Yunan \\
\hline S11 & A & $\begin{array}{l}\text { Haikou, } \\
\text { Hainan }\end{array}$ & S27 & C & $\begin{array}{l}\text { Zhongshan, } \\
\text { Guangdong }\end{array}$ & $\mathrm{S} 43$ & D & Puer, Yunan \\
\hline S12 & $\mathrm{A}$ & $\begin{array}{l}\text { Hainan, } \\
\text { Haikou }\end{array}$ & S28 & $\mathrm{C}$ & $\begin{array}{l}\text { Zhongshan, } \\
\text { Guangdong }\end{array}$ & $\mathrm{S} 44$ & $\mathrm{D}$ & $\begin{array}{l}\text { Xishuangbana } \\
\text { Yunnan }\end{array}$ \\
\hline $\mathrm{S} 13$ & A & $\begin{array}{l}\text { Haikou, } \\
\text { Hainan }\end{array}$ & S29 & C & $\begin{array}{l}\text { Zhongshan, } \\
\text { Guangdong }\end{array}$ & S45 & D & Puer, Yunan \\
\hline S14 & $\mathrm{A}$ & $\begin{array}{l}\text { Haikou, } \\
\text { Hainan }\end{array}$ & S30 & $\mathrm{C}$ & $\begin{array}{l}\text { Zhongshan, } \\
\text { Guangdong }\end{array}$ & S46 & $\mathrm{D}$ & $\begin{array}{l}\text { Zhongshan, } \\
\text { Guangdong }\end{array}$ \\
\hline S15 & B & $\begin{array}{l}\text { Huazhou, } \\
\text { Guangxi }\end{array}$ & S31 & $\mathrm{C}$ & $\begin{array}{l}\text { Huazhou, } \\
\text { Guangxi }\end{array}$ & S47 & $\mathrm{D}$ & Haikou, Hainan \\
\hline S16 & B & $\begin{array}{l}\text { Zhongshan, } \\
\text { Guangdong }\end{array}$ & S32 & $\mathrm{C}$ & Haikou, Hainan & $\mathrm{S} 48$ & $\mathrm{D}$ & Beihai, Guangxi \\
\hline
\end{tabular}

\subsection{Sample Preparation}

All samples were ground into fine powder using a grinder. The 95/5 $(v / v)$ ethanol/water extraction were acquired by thermal reflux. Supernatant were filtered through $0.45 \mathrm{um}$ filters and stored at $4{ }^{\circ} \mathrm{C}$ before LC-MS analysis. $10 \mathrm{~mL}$ ethanol extracts were vaporized at $50{ }^{\circ} \mathrm{C}$ water bathing and re-dissolved in ethyl acetate and used for GC-MS analysis after desiccation and filtration.

\subsection{GC-MS Analysis}

GC-MS were performed using a GC coupled with quad-mass spectrometer (GC2010, Shimadzu, Kyoto, Japan). Analytic conditions were as follows: detector temperature, $230{ }^{\circ} \mathrm{C}$; injection port temperature, $250{ }^{\circ} \mathrm{C}$; column DB-5HT $(30 \mathrm{~m} \times 0.25 \mathrm{~mm}$, Agilent Technologies AG, Waldbronn, Germany). Sample size: $1 \mu \mathrm{L}$. The split ratio was 1:20, with helium as the carrier gas at a flow rate of $0.5 \mathrm{~mL} / \mathrm{min}$. The oven temperature program was as follows: $90^{\circ} \mathrm{C}$ for $1 \mathrm{~min} ; 90^{\circ} \mathrm{C}$ to $150{ }^{\circ} \mathrm{C}$ with a gradient of $2{ }^{\circ} \mathrm{C} / \mathrm{min}$ and held at $150^{\circ} \mathrm{C}$ for $5 \mathrm{~min} ; 150{ }^{\circ} \mathrm{C}$ to $280{ }^{\circ} \mathrm{C}$ with a gradient of $2{ }^{\circ} \mathrm{C} / \mathrm{min}$ and held at $280^{\circ} \mathrm{C}$ for $10 \mathrm{~min}$. The TIC was acquired in full scanning mode (mass range $50-500 \mathrm{~m} / \mathrm{z}$ ). A solvent delay of $2.5 \mathrm{~min}$ was used. The alkaline mixture standard (C9-C40) were analyzed by the same program in GC-MS. The identification of components were assigned by the software GC-MS Postrun analysis (Shimadzu, Kyoto, Japan) by searching MS data and retention indices in National Institute of Standards and Technology (NIST, Washington DC, USA) and referring to previous reports [30,31]. Areas were recorded for all detectable peaks. Areas of each identified peaks were quantified and their proportions were calculated by GC-MS Postrun analysis (Shimadzu, Kyoto, Japan). Proportions of 
total sesquiterpenoid, PECs and other chemicals were separately calculated by sum of proportions of chemicals belonging to sesquiterpenoid, PECs and other chemicals.

\subsection{LC-MS/MS Analysis}

LC-MS were performed using an ultra-high performance liquid chromatography (UPLC) system (1290 Infinity II, Agilent Technologies, Singapore) coupled with triple quad-mass spectrometer (6420 Triple Quad, Agilent Technologies, Singapore). Data acquisition and LC-MS data analysis were carried out by MassHunter Acquisition ${ }^{\circledR}$, MassHunter Qualitative Analysis ${ }^{\circledR}$ (8.00, Agilent Technologies, Santa Clara, CA, USA). A C18 column $(100 \times 2.1 \mathrm{~mm}, 1.7 \mu \mathrm{m}$, Phenomenex, Washington, USA) was used for chromatographic separations. The mobile phases were acetonitrile (A) and $0.1 \%$ formic acid-water (B). A gradient elution was used: $10-20 \%$ at $0-5 \mathrm{~min}, 20-25 \%$ at $5-9 \mathrm{~min}, 25-30 \%$ at $9-12 \mathrm{~min}, 30-35 \%$ at $12-16 \mathrm{~min}, 35 \%$ at $16-16.3 \mathrm{~min}, 35-42 \%$ at $16.3-19 \mathrm{~min}, 42-60 \%$ at $19-28 \mathrm{~min}$, $60-95 \%$ at $28-29 \mathrm{~min}, 95-10 \%$ at $29-30 \mathrm{~min}$. The flow rate was $0.3 \mathrm{~mL} / \mathrm{min}$ and the injection volume was $0.5 \mathrm{uL}$. The vaporizer gas was $\mathrm{N}_{2}$ at $350{ }^{\circ} \mathrm{C}$ and 60 psi. Positive ions were analyzed under scan, product ion, and multi reaction monitoring (MRM) model separately. In scan model, $\mathrm{m} / \mathrm{z}$ ranged at 100-600 with fragmentor at $135 \mathrm{v}$. Collision energy (CE) for each precursor ions were optimized according the abundance of characteristic product ions. The molecular formula and possible structure were deduced according the precursor ion and product ions $\mathrm{m} / \mathrm{z}$ according to the standards and previous research $[9,32]$. Areas of each identified peaks were quantified and their proportions were calculated by MassHunter Qualitative Analysis ${ }^{\circledR}$. Proportions of total THEPECs, EPECs, DEPECS, and FPECs were separately calculated by sum of proportions of chemicals identified to THEPECs, EPECs, DEPECS, and FPECs.

\subsection{Data Processing and Analysis}

Raw LC-MS data were converted tomzXML format using MSconvert tools (Version 3,64 bit, proteowizard, Palo Alto, CA, USA). Raw GC-MS data were converted to mzXML format using GC-MS Postrun analysis (Shimadzu, Kyoto, Japan). Preprocessing of MS data including peak picking, peak grouping, retention time (RT) correction, and integration was performed using the XCMS implemented with R software (Version 3.5, University of Auckland, New Zealand). Each ion was identified by the $\mathrm{RT}$ and $m / z$ data. Intensities of each peak were recorded and a three-dimensional matrix containing arbitrarily assigned peak indices and ion intensity information was generated. The intensities of each ions identified were normalized and the quantitative data were analyzed by several unsupervised methods and supervised methods in R. PCA (principle components analysis) was used for multivariate exploration of clusters and trends among the observation. Feature selection were performed by Boruta package in $\mathrm{R}$.

Author Contributions: Conceptualization, T.Y. and G.L.; methodology, T.Y.; software, T.Y.; validation, T.Y., S.Y. and Y.C.; formal analysis, T.Y.; investigation, T.Y. and S.Y.; resources, G.L.; data curation, T.Y.; writing-original draft preparation, T.Y.; writing-review and editing, T.Y. and Q.W.; project administration, T.Y.; funding acquisition, T.Y.

Funding: This research was funded by National Nonprofit Special Fund for Fundamental Research from Chinese Academy of Forestry, grant number CAFYBB2018SY033.

Acknowledgments: In this section you can acknowledge any support given which is not covered by the author contribution or funding sections. This may include administrative and technical support, or donations in kind (e.g., materials used for experiments).

Conflicts of Interest: The authors declare no conflict of interest. The funders had no role in the design of the study; in the collection, analyses, or interpretation of data; in the writing of the manuscript, or in the decision to publish the results. 


\section{References}

1. Kristanti, A.N.; Tanjung, M.; Aminah, N.S. Review: Secondary Metabolites of Aquilaria, a Thymelaeaceae Genus. Mini. Rev. Org. Chem. 2018, 15, 36-55. [CrossRef] [PubMed]

2. Ye, W.; Wu, H.; He, X.; Wang, L.; Zhang, W.; Li, H.; Fan, Y.; Tan, G.; Liu, T.; Gao, X. Transcriptome Sequencing of Chemically Induced Aquilaria sinensis to Identify Genes Related to Agarwood Formation. PLoS ONE 2016, 11, e0155505. [CrossRef] [PubMed]

3. Mohamed, R.; Wong, M.T.; Halis, R. Microscopic observation of 'gaharu' wood from Aquilaria malaccensis. Pertanika J. Sci. Technol. 2013, 36, 43-50.

4. Ye, W.; Zhang, W.; Liu, T.; Zhu, M.; Li, S.; Li, H.; Huang, Z.; Gao, X. iTRAQ-Based Quantitative Proteomic Analysis of Chemically Induced Aquilaria sinensis Provides Insights into Agarwood Formation Mechanism. Proteomics 2018, 18, e1800023. [CrossRef] [PubMed]

5. Putra, D.A.; Shiou, Y.L.; Diana, E.; Rozi, M. History and perspectives of induction technology for agarwood production from cultivated Aquilaria in Asia: A review. J. For. Res. 2019, 30, 1-11.

6. Chen, X.; Liu, Y.; Yang, Y.; Feng, J.; Liu, P.; Sui, C.; Wei, J. Trunk surface agarwood-inducing technique with Rigidoporus vinctus: An efficient novel method for agarwood production. PLoS ONE 2018, 13, e019811. [CrossRef]

7. Chen, X.; Zhu, X.; Feng, M.; Zhong, Z.; Zhou, X.; Chen, X.; Ye, W.; Zhang, W.; Gao, X. Relationship between Expression of Chalcone Synthase Genes and Chromones in Artificial Agarwood induced by Formic Acid Stimulation Combined with Fusarium sp. A2 Inoculation. Molecules 2017, 22, 686. [CrossRef]

8. Zhou, X.; Chen, L.Y.; Chen, X.D.; Zhong, Z.J.; Li, H.H.; Zhang, W.M.; Gao, X.X. Analysis and evaluation of chemically induced artificial agarwood. China J. Chin. Materia Med. 2017, 42, 3159-3166.

9. Li, Y.; Sheng, N.; Wang, L.; Li, S.; Chen, J.; Lai, X. Analysis of 2-(2-Phenylethyl)chromones by UPLC-ESI-QTOF-MS and Multivariate Statistical Methods in Wild and Cultivated Agarwood. Int. J. Mol. Sci. 2016, 17, 771. [CrossRef] [PubMed]

10. Chen, H.; Yang, Y.; Xue, J.; Wei, J.; Zhang, Z.; Chen, H. Comparison of compositions and antimicrobial activities of essential oils from chemically stimulated agarwood, wild agarwood and healthy Aquilaria sinensis (Lour.) gilg trees. Molecules 2011, 16, 4884-4896. [CrossRef] [PubMed]

11. Yang, J.; Dong, W.; Kong, F.; Liao, G.; Wang, J.; Li, W.; Mei, W.; Dai, H. Characterization and Analysis of 2-(2-Phenylethyl)-chromone Derivatives from Agarwood (Aquilaria crassna) by Artificial Holing for Different Times. Molecules 2016, 21, 911. [CrossRef] [PubMed]

12. Liu, Y.; Chen, J.; Qian, J.; Lin, H.; Sun, N.; Huang, Z. Evolutionary analysis and structural characterization of Aquilaria sinensis sesquiterpene synthase in agarwood formation: A computational study. J. Theor. Biol. 2018, 456, 249-260. [CrossRef] [PubMed]

13. Gao, Z.H.; Yang, Y.; Zhang, Z.; Zhao, W.T.; Meng, H.; Jin, Y.; Huang, J.Q.; Xu, Y.H.; Zhao, L.Z.; Liu, J.; et al. Profiling of microRNAs under wound treatment in Aquilaria sinensis to identify possible microRNAs involved in agarwood formation. Int. J. Biol. Sci. 2014, 10, 500-510. [CrossRef] [PubMed]

14. Dong, X.J.; Gao, B.; Feng, Y.; Liu, X.; Wang, J.; Wang, J.L.; Tu, P.; Wang, X. Production of 2-(2-phenylethyl) chromones in Aquilaria sinensis calli under different treatments. Plant. Cell Tissue Organ. Culture 2018, 135, 53-62. [CrossRef]

15. Liao, G.; Dong, W.H.; Yang, J.L.; Li, W.; Wang, J.; Mei, W.L.; Dai, H.F. Monitoring the Chemical Profile in Agarwood Formation within One Year and Speculating on the Biosynthesis of 2-(2-Phenylethyl)Chromones. Molecules 2018, 23, 1261. [CrossRef] [PubMed]

16. Liu, Y.Y.; Chen, D.L.; Wei, J.H.; Feng, J.; Zhang, Z.; Yang, Y.; Zheng, W. Four New 2-(2-Phenylethyl)chromone Derivatives from Chinese Agarwood Produced via the Whole-Tree Agarwood-Inducing Technique. Molecules 2016, 21, 1433. [CrossRef]

17. Liu, Y.; Chen, H.; Yang, Y.; Zhang, Z.; Wei, J.; Meng, H.; Chen, W.; Feng, J.; Gan, B.; Chen, X.; et al. Whole-tree agarwood-inducing technique: An efficient novel technique for producing high-quality agarwood in cultivated Aquilaria sinensis trees. Molecules 2013, 18, 3086-3106. [CrossRef]

18. Wang, S.; Yu, Z.; Wang, C.; Wu, C.; Guo, P.; Wei, J. Chemical Constituents and Pharmacological Activity of Agarwood and Aquilaria Plants. Molecules 2018, 23, 342. [CrossRef] 
19. Wang, M.R.; Li, W.; Luo, S.; Zhao, X.; Ma, C.H.; Liu, S.X. GC-MS Study of the Chemical Components of Different Aquilaria sinensis (Lour.) Gilgorgans and Agarwood from Different Asian Countries. Molecules 2018, 23, 2168. [CrossRef]

20. Shao, H.; Kong, F.D.; Wang, H.; Mei, W.L.; Dai, H.F. Qinanmer, a new compound from Chinese agarwood 'Qi-Nan' originating from Aquilaria sinensis. J. Asian. Nat. Prod. Res. 2017, 19, 935-940. [CrossRef]

21. Xia, B.; Li, J.; Yang, D.; Mei, W.; Ding, L.; Zhou, Y. A rapid and highly specific method to evaluate the presence of 2-(2-phenylethyl) chromones in agarwood by supercritical fluid chromatography-mass spectrometry. Eur. J. Mass Spectrom (Chichester) 2014, 20, 395-402. [CrossRef]

22. Ismail, S.N.; Maulidiani, M.; Akhtar, M.T.; Abas, F.; Ismail, I.S.; Khatib, A.; Ali, N.A.M.; Shaari, K. Discriminative Analysis of Different Grades of Gaharu (Aquilaria malaccensis Lamk.) via (1)H-NMR-Based Metabolomics Using PLS-DA and Random Forests Classification Models. Molecules 2017, 22, 1612. [CrossRef]

23. Kao, W.Y.; Hsiang, C.Y.; Ho, S.C.; Ho, T.Y.; Lee, K.T. Chemical Profiles of Incense Smoke Ingredients from Agarwood by Headspace Gas Chromatography-Tandem Mass Spectrometry. Molecules 2018, 23, 2969. [CrossRef] [PubMed]

24. Ahn, S.; Ma, C.T.; Choi, J.M.; An, S.; Lee, M.; Le, T.H.V.; Pyo, J.J.; Lee, J.; Choi, M.S.; Kwon, S.W.; et al. Adiponectin-Secretion-Promoting Phenylethylchromones from the Agarwood of Aquilaria malaccensis. J. Nat. Prod. 2019. [CrossRef] [PubMed]

25. Wang, S.; Wang, C.; Peng, D.; Liu, X.; Wu, C.; Guo, P.; Wei, J. Agarwood Essential Oil Displays Sedative-Hypnotic Effects through the GABAergic System. Molecules 2017, 22, 2190. [CrossRef]

26. Lee, S.Y.; Rozi, M.; Yin, Y.F.; Rasool, S.; Turjaman, M.; Gao, Z.H.; Subasinghe, S.M.C.U.P.; Tajuddin, S.N.; Kakino, M.; Karlinasari, L.; et al. Keeping up Appearances: Agarwood Grades and Quality. Agarwood, Tropical Forestry; Springer Science+ Business Media: Singapore, 2016; pp. 149-167.

27. Xu, Y.; Zhang, Z.; Wang, M.; Wei, J.; Chen, H.; Gao, Z.; Sui, C.; Luo, H.; Zhang, X.; Yang, Y.; et al. Identification of genes related to agarwood formation: Transcriptome analysis of healthy and wounded tissues of Aquilaria sinensis. Bmc Genomics. 2013, 14, 227. [CrossRef] [PubMed]

28. Zhang, Z.; Wei, J.; Han, X.; Liang, L.; Yang, Y.; Meng, H.; Xu, Y.; Gao, Z. The sesquiterpene biosynthesis and vessel-occlusion formation in stems of Aquilaria sinensis (Lour.) Gilg trees induced by wounding treatments without variation of microbial communities. Int. J. Mol. Sci. 2014, 15, 23589-23603. [CrossRef]

29. National Pharmacopoeia Committee. Pharmacopoeia of the People's Republic of China; Chinese Medical Science and Technology Press: Beijing, China, 2015; p. 185.

30. Mei, W.L.; Yang, D.L.; Wang, H.; Yang, J.L.; Zeng, Y.B.; Guo, Z.K.; Dong, W.H.; Li, W.; Dai, H.F. Characterization and determination of 2-(2-phenylethyl)chromones in agarwood by GC-MS. Molecules 2013, 18, 12324-12345. [CrossRef] [PubMed]

31. Yan, D.; Wong, Y.F.; Whittock, S.P.; Koutoulis, A.; Shellie, R.A.; Marriott, P.J. Sequential Hybrid Three-Dimensional Gas Chromatography with Accurate Mass Spectrometry: A Novel Tool for High-Resolution Characterization of Multicomponent Samples. Anal. Chem. 2018, 90, 5264-5271. [CrossRef]

32. Li, W.; Cai, C.H.; Dong, W.H.; Guo, Z.K.; Wang, H.; Mei, W.L.; Dai, H.F. 2-(2-phenylethyl)chromone derivatives from Chinese agarwood induced by artificial holing. Fitoterapia 2014, 98, 117-123. [CrossRef]

Sample Availability: Not available. 